\title{
Brief Introduction of Missile-satellite Cooperative Passive Location
}

\author{
Sijia $\mathrm{Li}^{1, \mathrm{a}}$, Fei $\mathrm{CaO}^{2, \mathrm{~b}}$ and Jiazhen Duan ${ }^{3, \mathrm{c}}$ \\ 1, 2, ${ }^{X}$ X'an Research Institute of High Technology, Xi'an, China \\ a13259798735@163.com, bcaofeimm@163.com, 'dbojue1989@163.com
}

\begin{abstract}
Keywords: Cooperative Location; Electronic Reconnaissance; Precise Strike; Electronic Reconnaissance; System Characteristics; Work Flow
\end{abstract}

\begin{abstract}
The longitudinal location precision of the missile borne electronic reconnaissance equipment is not enough. In order to strike the target more accurately, the method of missile-satellite cooperative passive location is proposed, based on the study of the concept of cooperative location, the hierarchy of the missile-satellite cooperative passive location system is divided, and the composition of the cooperative location system is researched according to different cooperative location level analysis. At last, the characteristics and work flow of the system are introduced.
\end{abstract}

\section{Introduction}

The battlefield situation is changing, in order to keep secret, the enemy radar and other radiation source target wartime frequency will change, and the position of the target will change at any time in the operation of anti-ship group, although the weapon system can detect the target position of the radiation source, but because of the small azimuth angle of the pitch azimuth, the longitudinal location precision of the system is not enough, at this point, if there are other reconnaissance platform cooperative operations, to make up for the shortage of weapon systems, and to achieve the goal of real-time precise location, it will improve the efficiency of combat greatly. As the main component of the electronic reconnaissance platform, the satellite has the advantages of large reconnaissance range and small environmental impact, with the development of aerospace electronic reconnaissance satellite technology, more satellites, more powerful functions, network reconnaissance, real-time communication can be realized, to provide the necessary conditions for missile and satellite cooperative localization. Therefore, it is of great significance in the practical combat to realize the precision strike and destroy of the target by improving the accuracy of locating the target to a great extent by using the cooperative location of the satellite and the missile under the condition of guaranteeing the time window.

\section{Development status of cooperative location}

\section{Foreign research status.}

Cooperative thinking in the nature and human society has a long history, as a scientific concept of synergetics in 1970s by the German physicist Hermann Haken formally proposed to form an independent scientific system. As one of the recognized twentieth Century system science the most important branch, the synergy theory and dissipative structure theory, catastrophe theory and known as the "new three" ${ }^{[1]}$. It provides new ideas and methods for the research of other scientific fields. In the fields of biology, computer intelligence and communication, cooperative thinking has attracted the attention of researchers, and its introduction has opened up a vast space for the research of their respective fields ${ }^{[2]}$.

In 1996, the Japanese scholar Ryo Kurazume proposed the concept of cooperative location ${ }^{\text {[3] }}$ mainly used for mobile robots real-time location information acquisition. For example, there are two sets of robots in the experiment. When one group moves, the other group remains stationary as a reference marker, and auxiliary groups move, and the two groups constantly exchange characters until all the robots reach the target position. Nowadays, the concept of cooperative location is 
extended from using the communication and measurement of nodes to support the location of a single node itself to the use of communication between nodes and measurement of the third party (cooperative target or non-cooperative target) to achieve location, that is, cooperative reconnaissance location or cooperative detection location.

In the military field, with the continuous development of the high-tech information warfare, the traditional missile and its operational model exposed more and more drawbacks. Therefore, more and more countries begin to pay close attention to and study the cooperation problem of missile weapon. In the 1970s, the United States put forward the concept of cooperative operation for the first time. At the same time, the development of the Russian "granite" supersonic anti-ship missiles to attack the target with the missile and bomb attack mode, but also reflects the concept of intelligent missile cooperative combat ${ }^{[4]}$. In 2002, the United States as the future combat system is an important part of "network fire" tactical missile system officially launched research, the system uses the data chain to achieve cooperative combat patrol attack missile and precision strike missiles. Raytheon delivered to the Navy 2000th Tomahawk cruise missile in flight to use satellite data to complete the chain coordination and guidance station information, but also reflects the concept of cooperative combat ${ }^{[5]}$ in 2010. In 2012, the Turin University of Technology, Italy, research team from the perspective of satellite location, cooperative location to the end of the mean acquisition time of the impact, and the location performance in different location specific scene analysis of cooperative localization algorithm, point to point network under the condition of satellite. In 2014, the EU CADDY system, through the "self-awareness diving collaborative project” to help human divers in hazardous areas to complete high-efficiency operations underwater pilot navigation system.

\section{Current domestic research.}

In China, the research on cooperative location ${ }^{[6]}$ is still relatively rare. The feasibility of cooperative location of two bombs has been studied by Peng Ruihui, Wang Guohong and Chen Shiju of Naval Aeronautical Engineering Institute ${ }^{[7]}$, the constraint conditions in two-dimensional space is derived and the location of the two together, the normal working conditions of the two missile information sharing and cooperative engagement were simulated, results show that, when the two missiles are far from the target, or far and near, the missile target location precision can be effectively improved. The algorithm of multi-ship cooperative location was studied by Xia Juncheng, Wang Chunbo, et al. ${ }^{[8,9]}$ of 723 Shipbuilding Industry Corporation. In this paper, the localization algorithm based on least square method and recursive weighted least squares method is discussed, and the localization algorithm is studied according to the number of platforms involved in location. Air-to-Air Missile Research Institute of China Dong Jipeng and Tang Dong ${ }^{[10]}$, proposed a target tracking algorithm based on two missiles' direction finding information. The least squares solution of the target position based on the principle of direction-finding cross-location is taken as the Kalman filter. This method can estimate the target velocity and avoid the problem that the position estimation variance is too large compared with the method of least-squares method, which can estimate the position and velocity of the target.Chinese Electronics Technology Group 38 Wan Hua ${ }^{[11]}$ explained the basic concept of multi-sensor cooperative location and work flow, to radar, acoustic, photoelectric sensor cooperative detection test as an example to verify the weighted least squares cooperative location of the typical algorithm, which validates the effectiveness of multi-sensor cooperative location algorithm to achieve the heterogeneous sensor complementary advantages. Air-to-Air Missile Research Institute of China Li Haijing ${ }^{[12]}$ for airborne radar jamming or passive detection system location accuracy under the condition of lack of air to air missile launch and guidance is not normal, put forward a kind of airborne passive radar and airborne remote anti-radiation missile network cooperative location method, this method can be accurate location of the radiation source target distance warning the plane of high value, provides an important guarantee for the realization of accurate attack and effective jamming. Shanghai Institute of Radio Equipment Wu Xiongjun and Ma Ning ${ }^{[13]}$ on multi-missile cooperative detection technology development research, in time domain, frequency domain, airspace and many other aspects of improving the missile weapon system in complex electromagnetic environment 
adaptability,and promote the development of cooperative detection technology. Zhang Lichuan and Ma Peng of Northwestern Polytechnical University ${ }^{[14,15]}$ introduced the cooperative navigation and location research of unmanned underwater vehicle. Through the development of cooperative navigation and location system, highlighting high efficiency, low cost, strong fault-tolerance and reconfigurable and other prominent advantages, the application prospect is broad. Beijing University of Aeronautics and Astronautics of the Wan Jiuqing ${ }^{[16]}$ and Nanjing University of Aeronautics and Astronautics Zhu Yanping ${ }^{[17]}$ on multi-UAV collaborative flight technology research, through multi-UAV collaborative attack strategy research, Man-machine attack when the actual combat effectiveness, improve combat capability. Zhang Jianhua of China University of Mining and Technology ${ }^{[18]}$ studied the multi-robot cooperative location technology to overcome the poor robustness and low efficiency of single-mobile robots, to ensure fast and accurate co-localization of multi-robot groups.

\section{Research on the concept of missile-satellite cooperative passive location}

\section{Concept of cooperative location.}

The basic idea of cooperative location is to utilize the advantages of reconnaissance and detection platform, to achieve the reconfiguration of different reconnaissance platforms on the same target through unified resource scheduling and collaborative task planning, and to obtain more accurate target information, to provide accurate protection.

Broadly speaking, the existing electronic reconnaissance systems in addition to individual independent reconnaissance equipment to carry out the task, the nature of the rest of the reconnaissance location method is the collaborative work of multiple devices. Such as multi station direction finding cross location system, time difference location system, etc., are multi station "collaborative" work, but only a single fixed mode of cooperation, and even can be said to be "rigid".

In this paper, a multi-platform cooperative passive location system is discussed, which uses 2 or more reconnaissance platforms to work together to achieve the purpose of the target location. That is, a platform-based, other platforms work together to carry out the task, is the coordination of action, which is cooperative location model is flexible, organized and variable.

\section{The classification of cooperative location.}

It can be divided into target coordination and regional coordination with the aim of cooperation. Target collaboration is the cooperative relationship determined by the reconnaissance and joint detection for the target, which has a strong purpose and information fusion processing is relatively easy. Regional collaboration is aimed at the designated area for reconnaissance and established cooperative relations, the general area of key areas, the target species in the region and the number of uncertain, so information fusion is difficult, often requiring manual intervention. This paper focuses on the goal of collaboration.

From the view of cooperative control domain, it can be divided into time domain cooperation and frequency domain cooperation. Time domain collaboration can be either for the same task of time collaboration, it can be complementary overlapping full-time domain coverage synergy, if the need for a region of the implementation of uninterrupted reconnaissance detection, then the need for each platform in the time window alternation Synergy; Frequency-domain cooperation can be either the same frequency band cooperation, it can be complementary overlapping full-frequency coverage coordination, especially in a region for reconnaissance detection, because the target number and frequency domain uncertainty, the need to be as wide as possible of the frequency domain coverage.

From the organization form of collaborative location system, the determination of cooperative relationship among the platforms can be divided into cooperative and self-organized coordination under the control of command center. There is no master-slave relationship among the platforms, and the status is equal. The self-organization collaboration can be divided into master-slave coordination and self-adaptive stochastic coordination.

The Concept of missile-satellite cooperative passive location.

Missile-satellite cooperative passive location system is composed of one missile and one satellite 
as the main body in order to achieve precision targets for the radiation source for the purpose of cooperative combat system. The core of the missile-satellite cooperative passive location technology is the colocation technology of heterogeneous electronic reconnaissance platform as shown in Fig.1. The system can be regarded as a combination of single target reconnaissance system, each subsystem according to the different radiation sources for reconnaissance location, each subsystem has the information processing platform, independent of the final, all the results from the comprehensive information processing platform for processing and then transmitted to the combat command system or weapon platform, complete destruction of the target cluster.

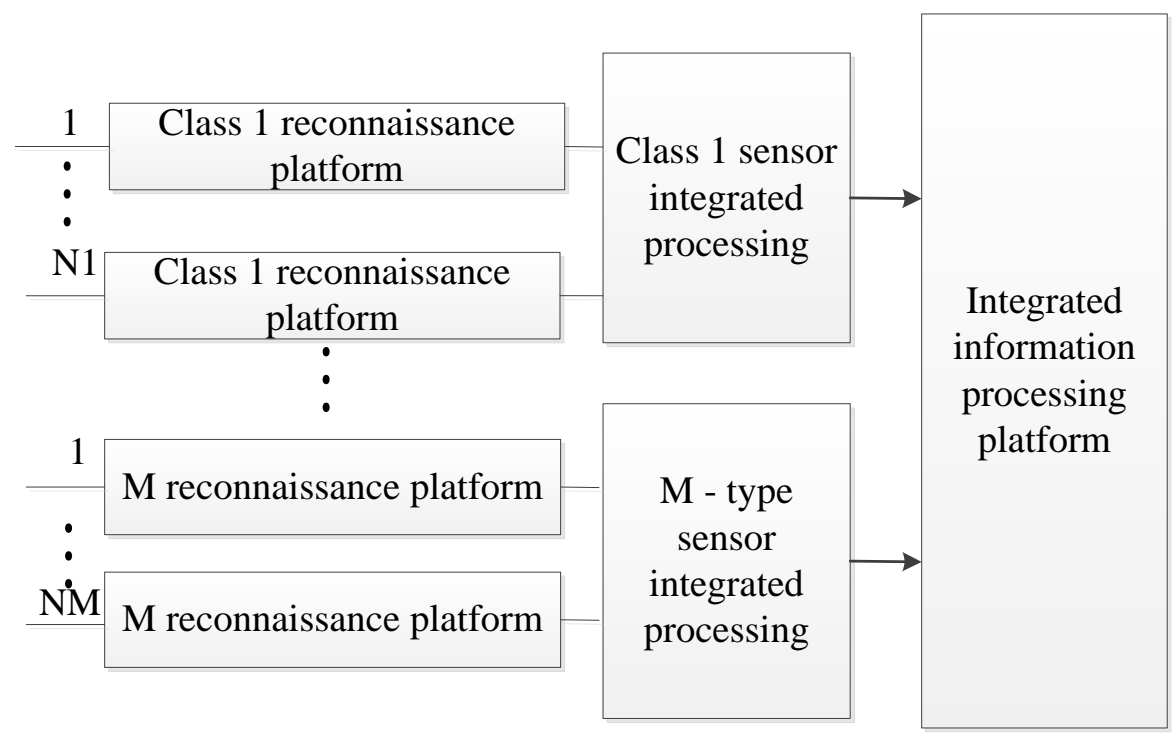

Fig.1 complex cluster target cooperative location system structure

As shown in Fig.2, the weapon system receives assistance in the process of operation in satellite reconnaissance information on the target, and their detection to information integration, to determine the target position, adjust their attitude, attack targets accurately.

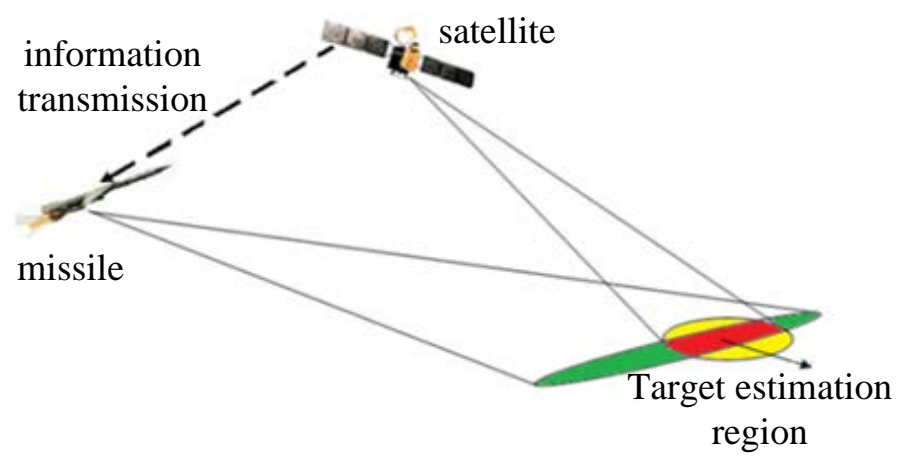

Fig.2 sketch map of missile-satellite cooperative passive location

\section{The hierarchical division of missile-satellite cooperative passive location}

According to the needs of collaborative tasks and the depth of participation in collaboration, missile-satellite cooperative passive location is divided into three cases in Table 1 . They are information-level collaboration, parameter-level collaboration and signal-level collaboration, due to the limited time window of satellite resources, it can not fully meet the whole process of missile and satellite real-time information exchange. 
Table 1 the feature contrast of three hierarchical divisions

\begin{tabular}{|c|c|c|c|c|}
\hline $\begin{array}{c}\text { collaborative } \\
\text { level }\end{array}$ & $\begin{array}{l}\text { collaborative } \\
\text { content }\end{array}$ & $\begin{array}{l}\text { achieve } \\
\text { difficulty }\end{array}$ & $\begin{array}{l}\text { location } \\
\text { accuracy }\end{array}$ & $\begin{array}{l}\text { Communication } \\
\text { bandwidth } \\
\text { requirements }\end{array}$ \\
\hline $\begin{array}{c}\text { information-level } \\
\text { collaboration }\end{array}$ & $\begin{array}{l}\text { the location } \\
\text { information of } \\
\text { the destination }\end{array}$ & simple & low & low \\
\hline $\begin{array}{l}\text { parameter-level } \\
\text { collaboration }\end{array}$ & $\begin{array}{l}\text { the parameter } \\
\text { information of } \\
\text { the target }\end{array}$ & medium & medium & medium \\
\hline $\begin{array}{c}\text { signal-level } \\
\text { collaboration }\end{array}$ & $\begin{array}{l}\text { the targ- } \\
\text { et signal is } \\
\text { intercepted }\end{array}$ & difficulty & high & high \\
\hline
\end{tabular}

Information-level collaboration: each detection platform has been data fusion on the basis of the target location which acquired, get more accurate target location information measurement process known as the information-level collaboration. Information-level collaboration is divided into two cases, one is complete information collaboration, that is, each platform has achieved the goal of location, but the accuracy is different, the collaboration is the use of reliable information fusion algorithm to provide different location information fusion platform to get a more accurate target location; The other is incomplete information coordination. In other words, the cooperative location system does not get the complete position information, but only intercepts some signals, or obtains several parameters. At this time, we can consider the joint fusion of parameters and position.

Parameter-level coordination: parameters including the arrival time of the pulse, the carrier frequency, pulse amplitude, pulse repetition interval, pulse width, pulse modulation characteristics, parameter-level coordination in order to obtain more accurate target position information, to avoid the various subsystems in data processing which introduces errors and generates noise.

Signal-level coordination: is the most difficult to achieve coordination, that is, the target platform will intercept the target signal directly transmitted to the main platform, do not make any processing or only for the initial processing, this can be the most accurate access to target information, but the attendant problem is that the communication data amount is large and the communication bandwidth is high. There are two kinds of signal-level coordination: the first is the direct cooperation of the sampled signal, the second is through the initial processing of signal data (such as the original pulse stream signal PDW) for coordination.

\section{The characteristics of missile-satellite cooperative passive location}

1. Diversification of information: Different reconnaissance detection platform of cooperative location system to provide a variety of system information, different types and different precision detection equipment can make the system get more information on the target, moreover, due to the limitations of their own detection range and working system ${ }^{[19]}$, a single platform is likely to detect blind spots and unrecognized targets. Multi-platforms can work together to reconcile reconnaissance time and spatial regions to compensate for the limitations of a single platform.

2. Low cost, highly cost effective: With the increasingly complex battlefield electromagnetic environment, the more challenges the enemy electronic reconnaissance will meet, compared to the development of a full-featured electronic reconnaissance equipment, it is easier and cost lower to build a cooperative location system which participate in collaboration between the platforms do not interfere with each other, the implementation of the task more fault-tolerant.

3. High precision, good reliability: the use of a single electronic reconnaissance platform for the implementation of electronic warfare mission risk is very large, once the battlefield by electromagnetic interference or enemy attack, the task is doomed to failure, but the use of 
cooperative location system can effectively avoid risks, even if the individual platform is damaged, other platforms can complete the task, with good robustness. And the joint aperture of each platform can increase the spatial resolution of the system. The information sharing can improve the location accuracy of the target, and these advantages are unattainable by a single electronic reconnaissance platform.

4. Limited communication timing ${ }^{[20]}$ : The biggest problem faced by low-Earth-orbiting satellites in practical applications is their limited coverage and time-varying nature ${ }^{[21]}$, which directly leads to a multi-frame data in a communication time. Transmission volume is small, while the high orbit satellite height is high, covering a large range, but the communication link transmission distance, transmission time, link loss, resulting in receiver power consumption, bulky, so according to the task requirements to choose different kinds of the electronic reconnaissance platforms.

\section{The working process of missile-satellite cooperative passive location system}

From the beginning to the end, the whole missile-satellite cooperative passive location task is divided into three steps, as shown in Fig.3:

1. System Construction: mainly by the command and control center. After the combat command, command and control center according to the known information of target object from the selection of collaborative comprehensive database, aiming at the complexity of the choice of target reconnaissance platforms and weapons composed of different cooperative location system.

2. Task planning: after the system is completed, the master-slave relationship between the cooperative members is determined. The communication links between the main members and other members are established.

3. Cooperative location: After the system is constructed, it determines the master-slave relationship among the cooperating members, establishes the communication link between the main member and other members, and assigns the task to finish the task planning.

4. Effect evaluation: The data generated in the whole coordination process and the damage effect to the target are sent back to the operational command system to complete the battlefield situation analysis.

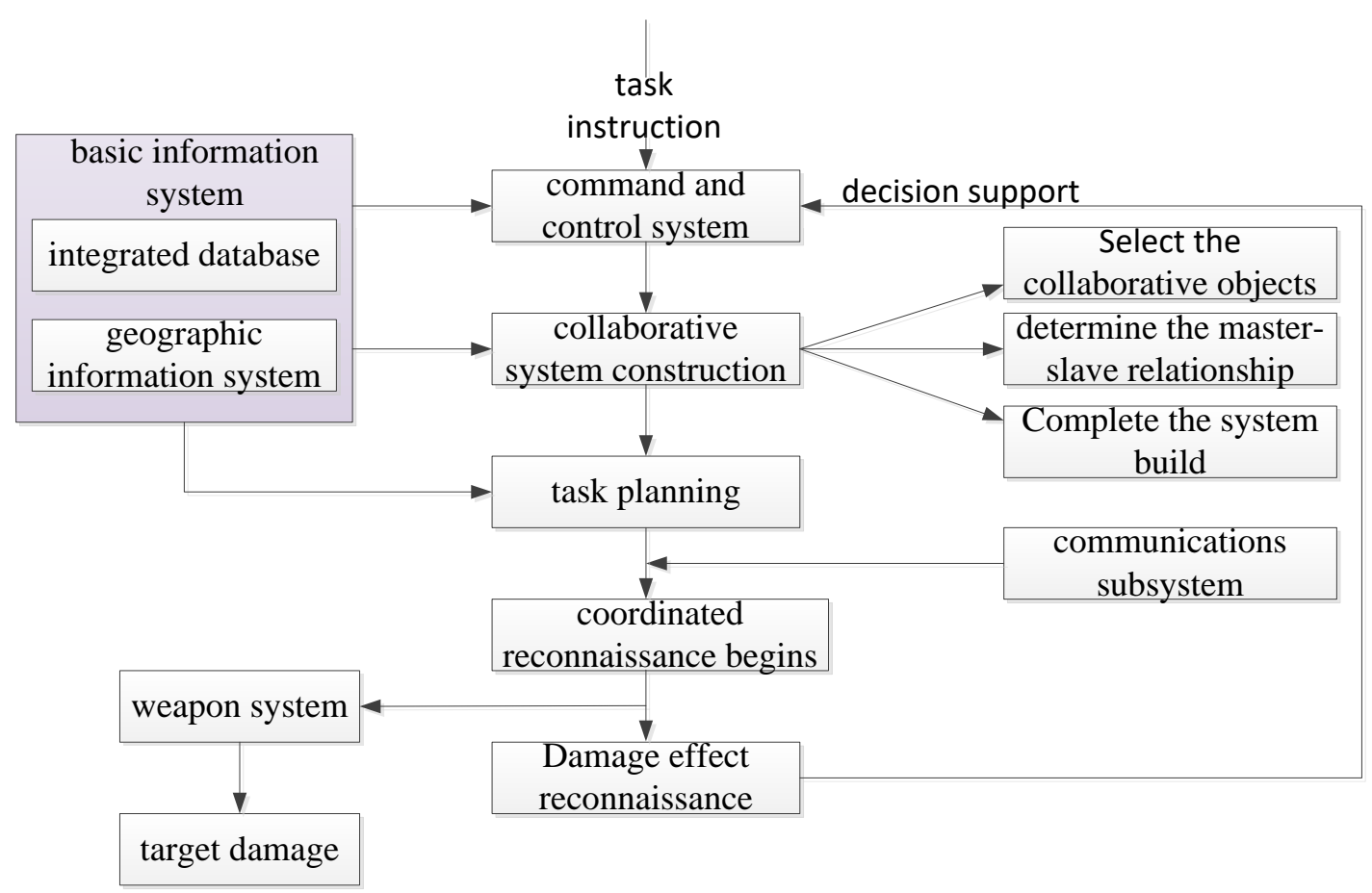

Fig.3 the working process of missile-satellite cooperative passive location system 


\section{Summary}

Aiming at the problem that the longitudinal location precision of the anti-radiation missile is not enough, this paper puts forward the method of missile-satellite cooperative passive location under the condition of ideal communication, in this paper, the concept of missile-satellite cooperative passive location, the characteristics and working process of missile-satellite cooperative passive location system are very important for practical combat. However, the battlefield situation is complicated. If we want to realize missile-satellite cooperative passive location, we must rely on the rich satellite resources and the reasonable task scheduling.

\section{References}

[1] Chen Kuining.The "new three” Enlightenment on dissipative structure theory, coordination theory and catastrophe theory [J]. Science and Technology Review, 1987(1):40-42.

[2] Liu Yaning, Li Guangxia, Chang Jiang, et al. Analysis of user location in cooperative localization [J]. Journal of Navigation and Location, 2014,2(3):20-24.

[3] KURAZUMER R, HIROSE S, NAGATA S, et al. Study on cooperative location system(basic principle and measurement experiment)[C]//Proceedings of 1996 IEEE International Conference on Robotics and Automation.. Minneapolis, 1996: 1421-1426.

[4] Su Ye. Design of three dimensional cooperative guidance law for anti-ship missile with angle constraint, Harbin: Harbin Institute of Technology, 2014. Tang Jiang, Xie Xiaofang, Sun Tao, et al. Research on coordinated attack strategy of intelligent anti-ship missile [J]. Winged Missile, 2012(11):57-61.

[5] He bing, Liu Gang, Xian Yong, et al. Controllable attack technology of cruise missile based on spatial information resources [J]. Information and Electronic Engineering, 2012, 10(5):538-541.

[6] Zhou Deyun, Hao Zhang, Qian Pan, et al. An improved range parameterized square root cubature information filter algorithm for multiuav cooperative passive location[C]//Proceeding of the IEEE International Conference on Information and Automation. Lijiang, 2015:1079-1084.

[7] Peng Yuehui, Wang Guohong, Chen Shiju, et al. The feasibility study of two cooperative location [J]. Journal of System SimulationHarbin Institute of Technology, 2006, 18(5):1118-1122.

[8] Xia Juncheng, Wang Chuanbo, Ni Tianquan. Algorithm of multi ship cooperative location technology [J]. Shipboard Electronic Countermeasure, 2008,31(3):71-73.

[9] Zhang Kai, Liu Yang. Research on passive location algorithm for slow target [J]. Radar Science and Technology 2014,12(3):291-296.

[10]Dong Jipeng, Tang Dong. Target tracking algorithm based on two missile cooperative location information [J]. Aviation Weapon, 2014(3):3-7.

[11]Wan Hua. Research on multi sensor cooperative localization technology [J]. Shipboard Electronic Countermeasure,2014,37(3):30-32.

[12] Li Haijing. Research on the technology of passive and passive localization of missile for long range air to air missile [J]. Modern Defense Technology, 2016, 44(1):16-21.

[13] Wu Qiongjun, Ma Ning. Review of multi missile cooperative detection [J]. Guidance and Fuze, 2016, 37(2):1-5.

[14]Zhang Lichuan, Xu Deming, Liu Mingyong, Yan Weisheng. Cooperative navigation and localization for multiple UUVs [J]. Journal of Ship and Ocean Engineering, 2009, 8(3):216-221.

[15]Ma Peng, Zhang Fubin, Xu Demin,Liu Shuqiang. Observability analysis of cooperative navigation system for multiple autonomous underwater vehicles based on condition number [J]. 
Acta Armamentarii, 2015,01.

[16]Wan Jiuqing, Bu Shaocong, Zhong Liping. Cooperative localization algorithm for UAV Based on hybrid dynamic belief propagation [J]. Journal of Beihang University, 2016,05:933-934.

[17]Zhu Yanping. Research on multi UAV cooperative attack strategy [D]. Nanjing: Nanjing University of Aeronautics \& Astronautics, 2012.

[18]Zhang Jianhua. Multi machine odor source localization in complex environment based on particle swarm optimization [D]. Beijing: China University of Mining and Technology, 2014.

[19]Zhao Hui. Research on target tracking algorithm based on multi-sensor information fusion [D]. Guangzhou: South China University of Technology, 2014.

[20]Yao Yao. Research on multi UUV cooperative localization technology [D]. Xi'an: Northwestern Polytechnical University, 2010.

[21]Cui Songqi. Research on Synchronization Technology of low orbit satellite communication system [D]. Beijing: Beijing Institute of Technology, 2015. 